\title{
Barreiras de potencial mapeadas por microscopia de força atômica em cerâmicas à base de $\mathrm{BaTiO}_{3}$ dopadas com lantânio
}

\author{
(Potential barriers mapping by atomic force microscopy in lanthanum \\ doped $\mathrm{BaTiO}_{3}$ based ceramics)
}

\author{
M. W. Mancini, P. I. Paulin Filho \\ Departamento de Engenharia de Materiais, Universidade Federal de S. Carlos \\ Rod. Washington Luiz, km 235, C.P. 676, S. Carlos, SP 13565-905 \\ mancini@iris.ufscar.br
}

\begin{abstract}
Resumo
Cerâmicas à base de titanato de bário $\left(\mathrm{BaTiO}_{3}\right)$ dopadas com $0,4 \%$ em mol de La e $150 \mathrm{ppm}$ de $\mathrm{Mn}$ foram investigadas por microscopia de força atômica no modo de monitoramento de gradientes de campo elétrico, denominado microscopia de força elétrica, para revelar as barreiras de potencial nos contornos de grão. Estas barreiras elétricas são associadas com o abrupto aumento na resistividade elétrica que ocorre a partir da temperatura de transição ferroelétrica- paraelétrica em $\mathrm{BaTiO}_{3}$ dopado com pequenas quantidades de doadores. Mostramos que para a composição utilizada neste estudo a largura das barreiras elétricas é da ordem de $350 \mathrm{~nm}-400 \mathrm{~nm}$, presentes nos contornos de grãos de tamanho médio de $1 \mu \mathrm{m}$.

Palavras-chave: titanato de bário, condutividade elétrica, barreiras de potencial, efeito PTC, microscopia de força atômica, microscopia de força elétrica.
\end{abstract}

\begin{abstract}
Barium titanate (BaTiO $)_{3}$ based ceramics doped with La as donor (0.4at\%t) and Mn as acceptor (150 ppm) were investigated through atomic force microscopy operating in electric force microscopy mode in order to reveal the potential barriers at grain boundaries. These barriers are associated with the steep rise in the resistivity above the ferroelectric-paraelectric phase transition temperature in $\mathrm{BaTiO}_{3}$ doped with small amounts of donor impurities. We show here that for such a composition the potential barrier width are of the order of 350-400 nm, present at grain boundaries of grains with size around $1 \mu \mathrm{m}$.

Keywords: barium titanate, electric conductivity, potential barriers, PTC effect, atomic force microscopy, electric force microscopy.
\end{abstract}

\section{INTRODUÇÃO}

O titanato de bário $\left(\mathrm{BaTiO}_{3}\right)$ é um material cerâmico com variadas aplicações em diversas áreas devido as suas propriedades elétricas e ópticas. Na área de dispositivos ópticos encontra aplicações devido a propriedades de birrefringência, e na área de dispositivos eletro-eletrônicos é utilizado na composição de resistores não-lineares (termistores PTC) e em capacitores devido a sua alta permissividade elétrica [1].

Termistores $\mathrm{PTC}$ à base de $\mathrm{BaTiO}_{3}$ são empregados nas mais diversas aplicações, tais como em sensores de temperatura, circuitos desmagnetizadores de tubos de televisores, secadores de cabelo, fornos de microondas, motores de partida, bem como em proteção de circuitos, etc.. O termo PTC refere-se a resistores cuja resistividade é sensível à temperatura e são geralmente obtidos a partir de $\mathrm{BaTiO}_{3}$ dopado com pequenas quantidades (de 0,1 a 0,3 at.\%) de doadores trivalentes (que substituem o $\mathrm{Ba}^{2+}$ ) ou pentavalentes nos sítios $\mathrm{Ti}^{4+}$ para introdução de semicondutividade do tipo $\mathrm{n}$ ao titanato de bário, que em seu estado puro é altamente resistivo (resistividade $>10^{10} \mathrm{ohm} \cdot \mathrm{cm}$ ). Aditivos como $\mathrm{Cu}$, $\mathrm{Fe}, \mathrm{Mn}, \mathrm{Ru}$ e V são geralmente adicionados a fim de obterse uma amplificação do efeito, e atuam como aceitadores de elétrons [2]. O valor da resistência elétrica nos PTCs elevase pronunciadamente em torno da temperatura de Curie, $\mathrm{T}_{c}$, correspondente à transição de fase ferroelétrica-paraelétrica (estrutura tetragonal e cúbica, respectivamente) [1-4].

Vários trabalhos são encontrados na literatura reportando o estudo de diferentes dopantes, no caso dos termistores PTCs, visando a otimização elétrica destas cerâmicas eletrônicas e estudando os mecanismos de condução nestes dispositivos. Contudo, tanto os mecanismos de condução, como o efeito PTC ainda não são totalmente compreendidos até o presente momento. O efeito PTC relaciona-se intimamente à microestrutura, e está associado à formação de duplas barreiras elétricas (Fig. 1) dependentes da temperatura nas regiões de contornos de grão da cerâmica [2-4]. 


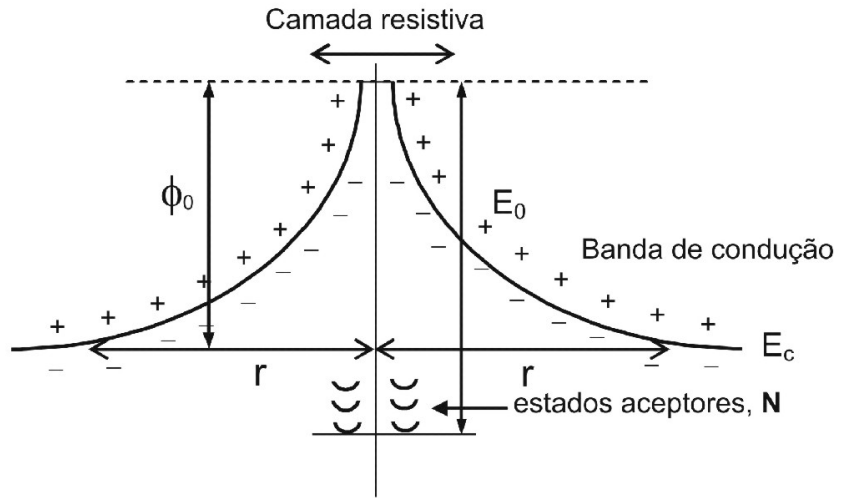

Figura 1: Representação esquemática de barreira de potencial presente em regiões de contornos de grãos em PTCs à base de $\mathrm{BaTiO}_{3}$ dopado com impurezas doadoras. $\mathrm{N}$ representa a densidade de estados aceitadores junto à camada resistiva no contorno de grão $[3,4]$.

[Figure 1: Schematic representation of the potential barrier present at grain boundary regions in donor-doped $\mathrm{BaTiO}_{3}$ based PTCs. $N$ is the density of acceptor states placed at the resistive layer at grain boundaries [3, 4].]

A formação da camada resistiva presente nas regiões de contornos de grão, que envolvem os grãos semicondutores, resulta na criação de barreiras de potencial devido ao balanceamento dos níveis de Fermi. Cargas espaciais superficiais surgem nas vizinhanças dos contornos de grãos devido ao fluxo de elétrons provenientes do interior dos grãos em direção aos contornos. Durante o resfriamento da amostra a partir de tratamentos a altas temperaturas, em atmosferas oxidantes ou a baixas taxas de resfriamento, ocorre a segregação de doadores e aceitadores para junto das regiões de contornos de grãos. Impurezas aceitadoras e vacâncias de titânio, bário e oxigênio, bem como elétrons livres e buracos podem atuar como estados aceitadores. A densidade de estados aceitadores presentes junto à camada resistiva depende fortemente de parâmetros de processamento, tais como pressão parcial de oxigênio, temperaturas de sinterização e de tratamento térmico, taxas de resfriamento, além de outros fatores cinéticos [5].

A altas temperaturas $\left(\mathrm{T}>\mathrm{T}_{\mathrm{C}}\right)$ o aumento da barreira de potencial previne o fluxo de portadores de carga, explicando a alta resistividade observada acima da temperatura de transição ferroelétrica. Para $\mathrm{T}<\mathrm{T}_{\mathrm{C}}$, a barreira é total ou parcialmente neutralizada pela polarização dos contornos relativa a fase ferroelétrica do material.

No $\mathrm{BaTiO}_{3}$ dopado com quantidades moderadas de impurezas doadoras (0,1 a 0,3 mol\%) [2], o aumento da condutividade a baixas temperaturas $\left(\mathrm{T}<<\mathrm{T}_{\mathrm{C}}\right)$ é atribuído ao excesso de elétrons de condução gerados pela incorporação dos doadores na rede cristalina $[3,4]$. Contudo, acima de 0,3 at.\%, a dependência da condutividade elétrica permanece não muito bem compreendida [6-13].

Embora existam diversas explicações para a transição do comportamento condutor para isolante, muitos deles não propõem mecanismos de formação ou a natureza das armadilhas. A explicação dada por Heywang [3, 4] para esta transição baseia-se em termos da compensação iônica de doadores por armadilhas aceptoras duplamente carregadas sem dar, contudo a natureza de tais armadilhas. Jonker [8] também sugeriu compensação de vacâncias sem propor o mecanismo. Wernicke [13] propôs a formação de vacâncias de Ba nas superfícies e contornos de grãos que difundem para o interior dos grãos de modo a compensar os doadores, contudo o modelo não considera a inibição do crescimento de grão. Além disso, o mecanismo de formação de vacâncias de Ba não é bem compreendido. Apesar da existência de vários modelos com o intuito de considerar a alta resistividade observada em $\mathrm{BaTiO}_{3}$ altamente dopado, nenhum deles foi devidamente comprovado.

A dependência entre propriedades elétricas e a microestrutura é bem documentada na literatura [9, 13-17] e discutida no trabalho de Desu e Payne [6] onde abordam a inter-relação entre composição química, desenvolvimento da microestrutura e propriedades elétricas, ressaltando a dependência do tamanho de grãos e da condutividade elétrica com a quantidade do dopante doador em $\mathrm{BaTiO}_{3}$ semicondutor. Estes autores mostram que as propriedades elétricas são fortemente dependentes da distribuição heterogênea de defeitos e da segregação interfacial do dopante doador, como também do aceitador.

Neste artigo apresentamos imagens das barreiras elétricas presentes na composição PTC descrita por $\mathrm{Ba}_{1-\mathrm{x}} \mathrm{La}_{\mathrm{x}} \mathrm{TiO}_{3}$, com $\mathrm{x}=0,004$. $\mathrm{O}$ imageamento das barreiras elétricas foi feito através de microscopia de força elétrica (EFM), utilizando a técnica de AFM.

A técnica de EFM, baseada no monitoramento dos gradientes verticais da força elétrica entre um cantilever e a amostra, é utilizada para mapear o gradiente vertical da força elétrica entre a ponta e a amostra versus as coordenadas $(\mathrm{x}, \mathrm{y})$, onde é criada uma imagem tridimensional, podendo também ser utilizada para a obtenção de distribuições de carga superficiais.

O mapeamento dos gradientes de força elétrica sobre a amostra é realizado através do monitoramento da variação $(\Delta f)$ na freqüência de ressonância $f_{0}$ do cantilever, que é ocasionada pelas interações eletrostáticas ponta-amostra, conforme representado esquematicamente na Fig. 2.

Além de variações na freqüência, os dados de EFM podem ser detectados também através de variações na fase ou amplitude de oscilação da ponta de prova, sendo a última técnica de detecção desaconselhável por efeito de possível presença de interferência óptica. Além disso, do ponto de vista da eletrônica envolvida na detecção do sinal de EFM, a detecção de modificações na freqüência e/ou fase são muito mais rápidas do que variações na amplitude.

Nesta técnica de AFM em que são medidas as variações no gradiente do campo elétrico sobre a amostra, a mesma pode ser condutora, não-condutora ou mista. No caso de amostras não condutoras ou fracamente condutoras é necessária aplicação de tensões externas, à ponta de prova ou à amostra, para aumentar o contraste elétrico.

As medidas de EFM são realizadas em dois passos (lift mode). Na primeira linha de varredura a topografia 


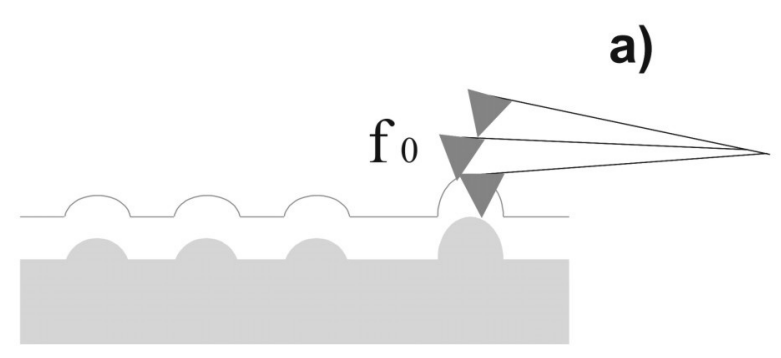

b)

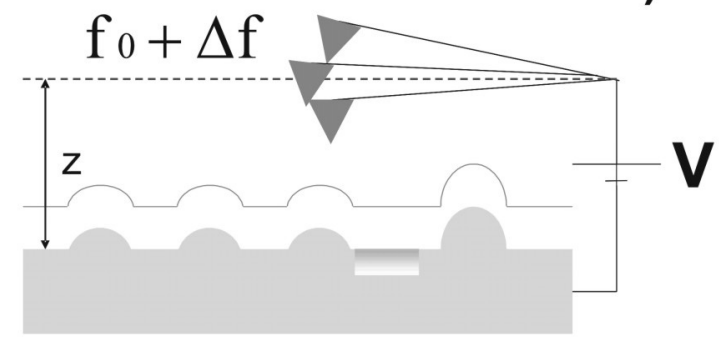

Figura 2: Esquema mostrando o princípio de funcionamento de microscopia de força elétrica (EFM) utilizando AFM, baseada na técnica de duas passagens (lift mode). Na primeira passagem são registrados os dados de topografia em modo contato intermitente (tapping mode). Na segunda, a varredura é feita a uma altura fixa, $\mathrm{z}$, da amostra em modo não-contato, seguindo o perfil de topografia obtido na primeira linha de varredura, enquanto são registrados os dados da força elétrica ocasionada à ponta pela amostra, enquanto os efeitos de topografia são minimizados.

[Figure 2: EFM scheme using AFM, based on the two passages technique (lift mode). In the former, the scan registers topography data in intermittent mode (tapping mode). In the later, electric force data are recorded while the cantilever is maintained at a fixed height from the sample, $z$, in non-contact mode, while minimizing topographic effects.]

é registrada em modo contato-intermitente. A seguir, o cantilever é levantado a uma altura fixa, tipicamente da ordem de $50-100 \mathrm{~nm}$, e então é realizada uma segunda varredura, em modo não-contato. Durante esta fase são adquiridos os dados de força elétrica.

Quando a amostra a ser estudada apresenta uma polarização macroscópica não é necessária aplicação de tensão, que no caso de amostras com fracos gradientes de campos elétricos superficiais se faz necessária para gerar o contraste necessário nas imagens, podendo ser aplicada tanto na ponta quanto na amostra. No caso da amostra investigada, contraste por EFM pode ser observado até mesmo para tensões aplicadas de $0 \mathrm{~V}$.

Neste trabalho, apresentamos resultados mostrando a formação de barreiras de potencial utilizando uma composição de $\mathrm{BaTiO}_{3}$ dopado com uma alta concentração de La como doador ( $0,4 \%$ atômico).

As técnicas utilizadas foram: microscopia de força atômica (AFM), operando em modo contato e modo contato intermitente, e microscopia de força elétrica (EFM) operada no modo EFG, que registra as variações no gradiente de campo elétrico.

Medidas da resistividade dc em função da temperatura foram feitas com o intuito de verificar e quantificar o efeito PTC nesta composição.

\section{MATERIAIS E MÉTODOS}

As cerâmicas estudadas foram preparadas a partir de $\mathrm{BaTiO}_{3}$ comercial de alta pureza (HPB TICON HPB - TAM Ceramics, Nyagara Falls) dopado com nitrato de lantânio hexa-hidratado, $\mathrm{La}\left(\mathrm{NO}_{3}\right)_{3} \cdot 6 \mathrm{H}_{2} \mathrm{O}$ (Aldrich Chemical Co., Inc.) na quantidade 0,4 mol\% manganês, na forma de sulfato de manganês mono-hidratado, $\mathrm{MnSO}_{4} \cdot \mathrm{H}_{2} \mathrm{O}$ (Mallinckrodt Chemical), foi adicionado na quantidade de $150 \mathrm{ppm}$. O pó de titanato de lantânio adicionado foi calcinado a $1100^{\circ} \mathrm{C}$ durante $2 \mathrm{~h}$.

Os dopantes foram introduzidos ao pó de titanato na forma de solução aquosa, e a mistura foi então homogeneizada por agitador magnético e moinho de bolas. As amostras foram conformadas em forma de pastilhas de aproximadamente 2,0 $\mathrm{mm}$ de altura e 10,0 $\mathrm{mm}$ de diâmetro através de prensagem uniaxial com carga de 1,5 ton. Antes de serem conformadas as amostras foram secas em estufa durante $2 \mathrm{~h}$. Após a conformação as pastilhas foram pesadas e medidas para o cálculo da densidade à verde. As amostras foram sinterizadas a $1350{ }^{\circ} \mathrm{C}$ durante um tempo de patamar de $2 \mathrm{~h}$ seguindo taxas de aquecimento de $5{ }^{\circ} \mathrm{C} / \mathrm{min}$. e de resfriamento de $10{ }^{\circ} \mathrm{C} / \mathrm{min}$, em forno elétrico (Lindberg-Blue M). Como aditivo de processamento foi utilizado álcool polivinílico (PVal, Hoescht) como ligante.

As amostras sinterizadas foram polidas com pasta de diamante de $1,0 \mu \mathrm{m}$ e $0,25 \mu \mathrm{m}$, e atacadas termicamente a $1250{ }^{\circ} \mathrm{C}$ durante 6 min para a revelação da microestrutura policristalina a ser investigada por AFM e EFM.

Eletrodos foram produzidos na amostra por aplicação de tinta prata, sendo que a amostra foi mantida em contato elétrico com o piezo de varredura (scanner) durante o imageamento por EFM. O cantilever é mantido aterrado durante todo o experimento.

Imagens de EFM foram obtidas com aplicação externa de tensão a amostra, através de uma fonte de corrente estabilizada (modelo MCP-303DI, Minipa).

As imagens de AFM (modo-contato, modo contato intermitente) e EFM foram obtidas à temperatura ambiente, com um microscópio de força atômica modelo Nanoscope IIIa (Digital Instruments). A ponta utilizada na obtenção das imagens de topografia em modo contato intermitente e de EFM foi a de modo não-contato NSG10 (MikroMasch), operando com freqüência de ressonância de $205,89 \mathrm{kHz}$.

Medidas de resistividade dc em função da temperatura foram feitas em um forno acoplado a um controlador de temperatura (Eurotherm) e a um multímetro HewlettPackard modelo 3457A.

\section{RESULTADOS E DISCUSSÃO}

O efeito PTC nesta amostra é mostrado na Fig. 3, que apresenta a variação da resistividade com a temperatura, com um efeito PTC de baixa magnitude. A resistividade medida na temperatura ambiente é igual a $13 \mathrm{kOhm} \cdot \mathrm{cm}$. 


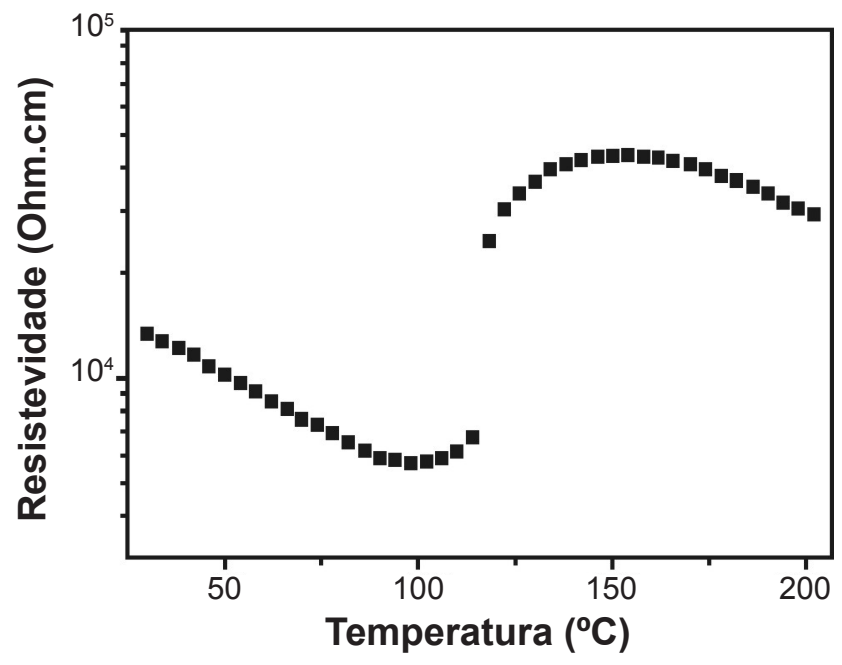

Figura 3: Curva típica de resistividade vs. temperatura para a composição $\mathrm{Ba}_{0,006} \mathrm{La}_{0,004} \mathrm{TiO}_{3}$.

[Figure 3: Typical resistivity vs. temperature curve for the $\mathrm{Ba}_{0.006} \mathrm{La}_{0.004} \mathrm{TiO}_{3}$ composition.]

No intervalo compreendido entre a temperatura ambiente e $100{ }^{\circ} \mathrm{C}$ ocorre um aumento de portadores de carga na banda de condução produzindo uma diminuição na resistividade com o aumento da temperatura (efeito NTC-negative temperature coefficient). A partir de $100{ }^{\circ} \mathrm{C}$ é observado o aumento da resistividade como função exponencial da temperatura, característica do efeito PTC. O aumento na resistividade neste intervalo é devido ao aumento na intensidade das barreiras de potencial dependentes da temperatura, que atuam impedindo a passagem dos portadores de carga do interior de um grão para o grão vizinho, e ocorre a partir da temperatura de mudança de estrutura tetragonal para cúbica. Para temperaturas superiores a $154^{\circ} \mathrm{C}$, o efeito NTC passa a

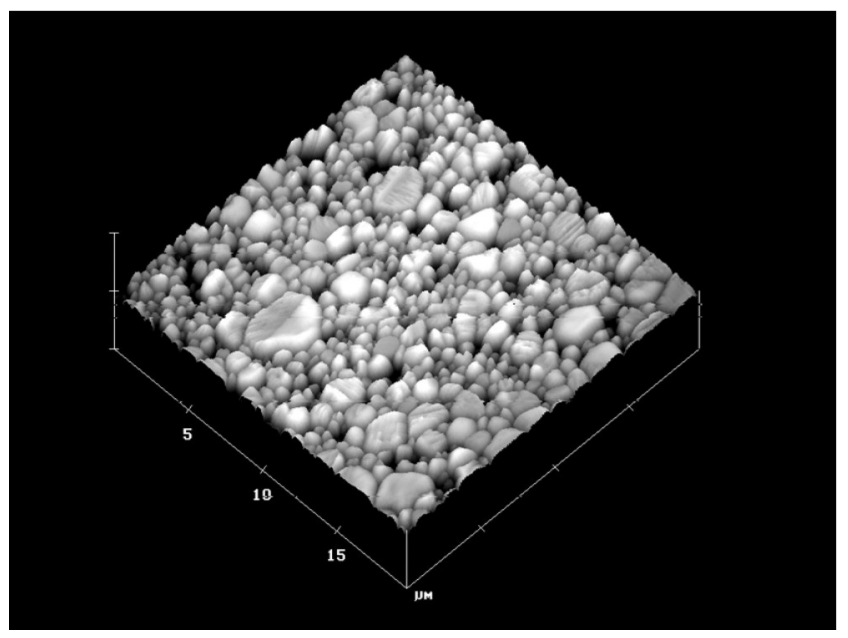

Figura 4: Imagem de topografia por AFM, em modo contato, da superfície de amostra de $\mathrm{BaTiO}_{3}$ dopado com La $(\mathrm{x}=0,004)$; $(20 \mu \mathrm{m} \times 20 \mu \mathrm{m} \times 1,5 \mu \mathrm{m})$.

[Figure 4: AFM topography image, obtained by contact mode, of the surface of La doped $\mathrm{BaTiO}_{3}(x=0.004) ;(20 \mu \mathrm{m} \times 20 \mu \mathrm{m}$ $\times 1.5 \mu \mathrm{m})$.]

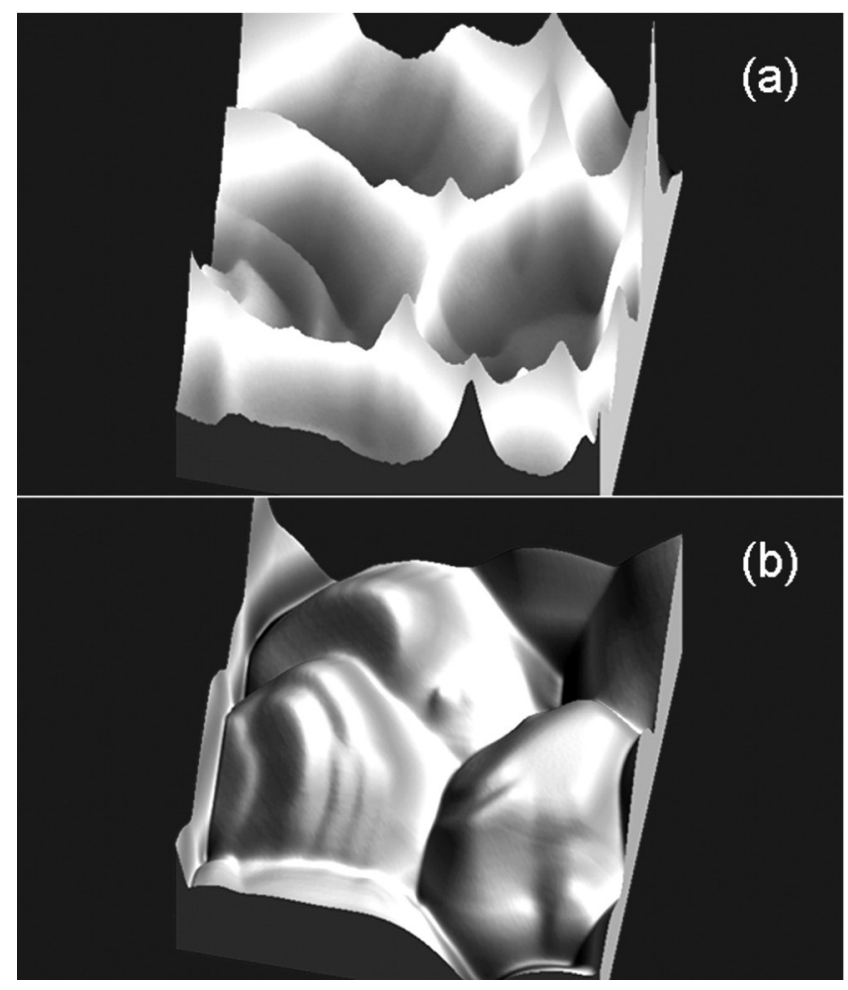

Figura 5: (a) Imagem de EFM obtida por monitoramento do gradiente de campo elétrico, aplicando-se $10 \mathrm{~V}$ à amostra $\mathrm{Ba}_{0,006} \mathrm{La}_{0,004} \mathrm{TiO}_{3}$. $(2 \mu \mathrm{m} \times 2 \mu \mathrm{m} \times 34 \mathrm{~Hz})$.

(b) Imagem de AFM (topografia) em modo contato intermitente (tapping mode) da área de varredura correspondente. $(2 \mu \mathrm{m} \times 2 \mu \mathrm{m}$ $\times 320 \mathrm{~nm}$ ).

[Figure 5: (a) EFM image obtained through electric field gradient monitoring with $10 \mathrm{~V}$ applied to the sample $\mathrm{Ba}_{0.006} \mathrm{La}_{0.004} \mathrm{TiO}_{3}$. $(2 \mu \mathrm{m}$ $\times 2 \mu m \times 34 \mathrm{~Hz}$ ).

b) AFM image (topography) in intermittent contact mode (tapping mode) of the corresponding region. $(2 \mu \mathrm{m} \times 2 \mu \mathrm{m} \times 320 \mathrm{~nm})$.]

predominar e a resistividade volta a diminuir com o aumento da temperatura.

As amostras apresentam baixa porosidade, requisito essencial para a realização da técnica de EFM. Os resultados obtidos por AFM mostram uma microestrutura com baixa porosidade, conforme mostra a imagem de topografia (Fig. 4). Observamos grãos com tamanho em torno de $1 \mu \mathrm{m}$ ou menores, e alguns raros grãos com crescimento anômalos, de 10 a $20 \mu \mathrm{m}$. Para esta composição, é teoricamente prevista uma microestrutura fina (grãos menores que $2 \mu \mathrm{m}$ ) e alta resistividade [6], em acordo com os resultados apresentados.

$\mathrm{Na}$ imagem de EFM mostrada na Fig. 5a, obtida com aplicação de $9 \mathrm{~V}$, a amostra, mantendo-se a ponta de prova aterrada, observa-se claramente a formação das barreiras de potencial nas regiões intergranulares. A Fig. 5b mostra a topografia da região correspondente.

A imagem de EFM (Fig. 5b) foi obtida com o equipamento de AFM operando na temperatura ambiente $\left(\mathrm{T}<<\mathrm{T}_{\mathrm{C}}\right)$, no regime de temperaturas em que o titanato de bário é semicondutor, mostrando que as barreiras não são 


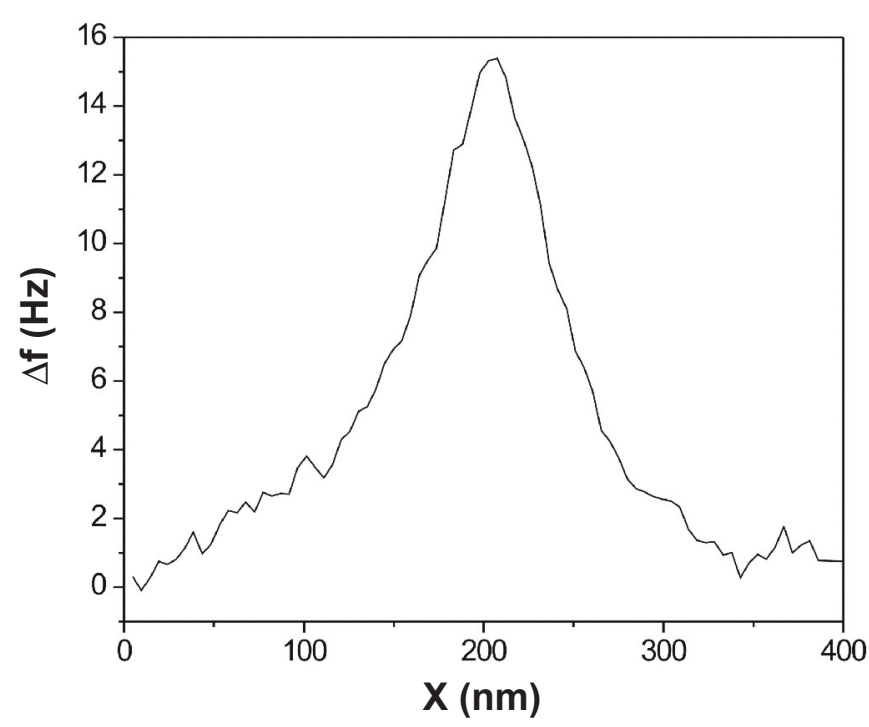

Figura 6: Perfil de intensidade típico de barreira elétrica presente na camada resistiva dos contornos de grão em $\mathrm{Ba}_{0,006} \mathrm{La}_{0,004} \mathrm{TiO}_{3}$, $\left(\mathrm{V}_{\mathrm{FFM}}=9 \mathrm{~V}\right)$.

[Figure 6: Typical intensity profile of the resistive grain boundary electric barrier in $\left.\mathrm{Ba}_{0.006} \mathrm{La}_{0.004} \mathrm{TiO}_{3},\left(V_{E F M}=9 \mathrm{~V}\right).\right]$

totalmente neutralizadas abaixo de $\mathrm{T}_{\mathrm{C}}$, ou seja, a polarização espontânea característica do estado ferroelétrico não é capaz de compensar completamente a carga superficial junto aos contornos de grãos, de modo que a resistividade, neste caso, ainda permanece governada pelas barreiras de potencial.

A largura média das barreiras elétricas determinadas através da análise das imagens de EFM é da ordem de $350 \mathrm{~nm}$, sendo que o tamanho médio dos grãos com esta composição cerâmica é da ordem de $1 \mu \mathrm{m}$. Assim, as barreiras elétricas resistivas assumem as dimensões dos grãos semicondutores, explicando parcialmente a alta resistividade a temperatura ambiente. Em amostras contendo 0,3at.\% de La, o tamanho de grão varia entre 10 e $30 \mu \mathrm{m}$. Neste caso, as larguras das barreiras elétricas não são comparáveis com as dimensões dos grãos.

A Fig. 6 mostra um perfil transversal típico das barreiras de potencial observadas, de acordo com o modelo de dupla barreira, proposto por Heywang [1, 2, 4], onde é observada uma largura de aproximadamente $350 \mathrm{~nm}$.

\section{CONCLUSÕES}

Imagens de microscopia de força elétrica mostrando barreiras de potencial elétrico em termistores PTC à base de $\mathrm{BaTiO}_{3}$ dopados com alta quantidade de impureza doadora $(0,4$ at.\% de La) foram obtidas usando microscopia de força elétrica (EFM). A composição investigada apresenta alta resistividade à temperatura ambiente $(13 \mathrm{kOhm} . \mathrm{cm})$ e ainda um efeito PTC, de baixa magnitude.

Imagens de EFM revelam a presença de barreiras de potencial nas regiões resistivas de contorno de grãos que envolvem os grãos semicondutores, conforme previstas teoricamente.
As imagens de EFM foram feitas em $\mathrm{BaTiO}_{3}$ semicondutor, à temperatura ambiente, portanto bem abaixo da temperatura de Curie, mostrando que as barreiras elétricas não são completamente compensadas pela polarização espontânea do regime ferroelétrico (fase tetragonal), revelando que a resistividade a baixas temperaturas também é determinada pelas barreiras de potencial.

A partir das imagens de EFM foram determinadas as larguras das barreiras elétricas, comparáveis com as dimensões dos grãos.

\section{AGRADECIMENTOS}

Ao MTA, Laboratório Nacional de Luz Síncrotron (LNLS), onde foram feitas as imagens de EFM, e ao CNPq pelo suporte financeiro.

\section{REFERÊNCIAS}

[1] Ceramic Materials for Electronics. Processing, Properties and Applications, $2^{\text {nd }}$ Ed., Ed. Relva C. Buchanan, Marcel Dekker Inc., New York (1991).

[2] S. B. Desu, D. A. Payne, Interfacial Segregation in Perovskites: IV, Internal Boundary Layer Devices, J. Am. Ceram. Soc. 73 (1990) 3416.

[3] W. Heywang, Barium Titanate as a Semiconductor with Blocking Layers, Solid State Electron. 3 (1961) 51.

[4] W. Heywang, Resistivity Anomaly in Doped Barium Titanate, J. Am. Ceram. Soc. 47 (1964) 484.

[5] S. B. Desu, The Nature of Defects in Semiconducting Barium Titanate, Ceram. Trans. 8, Ceramic Dielectrics: Composition, Processing and Properties, Ed. H. C. Ling, M. F. Yan, Am. Ceram. Soc., Westerville, OH (1990).

[6] S. B. Desu, D. A. Payne, Interfacial Segragation in Perovskites: III, Microestructure and Electrical Properties, J. Am. Ceram. Soc. 73 (1990) 3407.

[7] V. J. Tennery, R. L. Cook, Investigation of Rare-Earth Doped Barium Titanate, J. Am. Ceram. Soc. 44 (1961) 187.

[8] G. H. Jonker, Some Aspects of Semiconducting Barium Titanate, Solid State Electron. 7 (1964) 895.

[9] T. Fukami, H. Tsuchiya, Dependence of Resistivity on Donor Dopant Content in Barium Titanate Ceramics, Jpn J. Appl. Phys. 18 (1979) 735.

[10] I. Ueda, Oxidation Phenomena in Semiconducting $\mathrm{BaTiO}_{3}$, J. Phys. Soc. Jpn. 20 (1965) 546.

[11] H. Ueoka, The Doping Effects of Transition Elements on the PTC Anomaly of Semiconductive Ferroelectric Ceramics, J. Am. Ceram. Soc. 56 (1973) 351.

[12] G. V. Lewis, C. R. A. Catlow, R. E. W. Casselton, PTCR Effect in $\mathrm{BaTiO}_{3}$, J. Am. Ceram. Soc. 68 (1985) 555.

[13] R. Wernicke, The Kinectics of Equilibrium Restoration in Barium Titanate Ceramics, Philips Res. Rep. 31 (1976) 526.

[14] W. Heywang, Semiconducting Barium Titanate, J. Mater. Sci. 6 (1971) 1214.

[15] T. Murakami, T. Miyashita, M. Ankara, E. Sekine, Effect of Rare-Earth Ions on Electrical Conductivity of 
$\mathrm{BaTiO}_{3}$ Ceramics, J. Am. Ceram. Soc. 56 (1973) 294.

[16] W. Heywang, Structural Engeneering of Ferroelectrics, Ferroelectrics 49 (1983) 3.
[17] S. B. Desu, Grain Boundary Effects in Semiconducting $\mathrm{BaTiO}_{3}$, Euro-Ceram. 2 (1989) 2214.

(Rec. 16/05/2006, Ac. 09/02/2007) 